

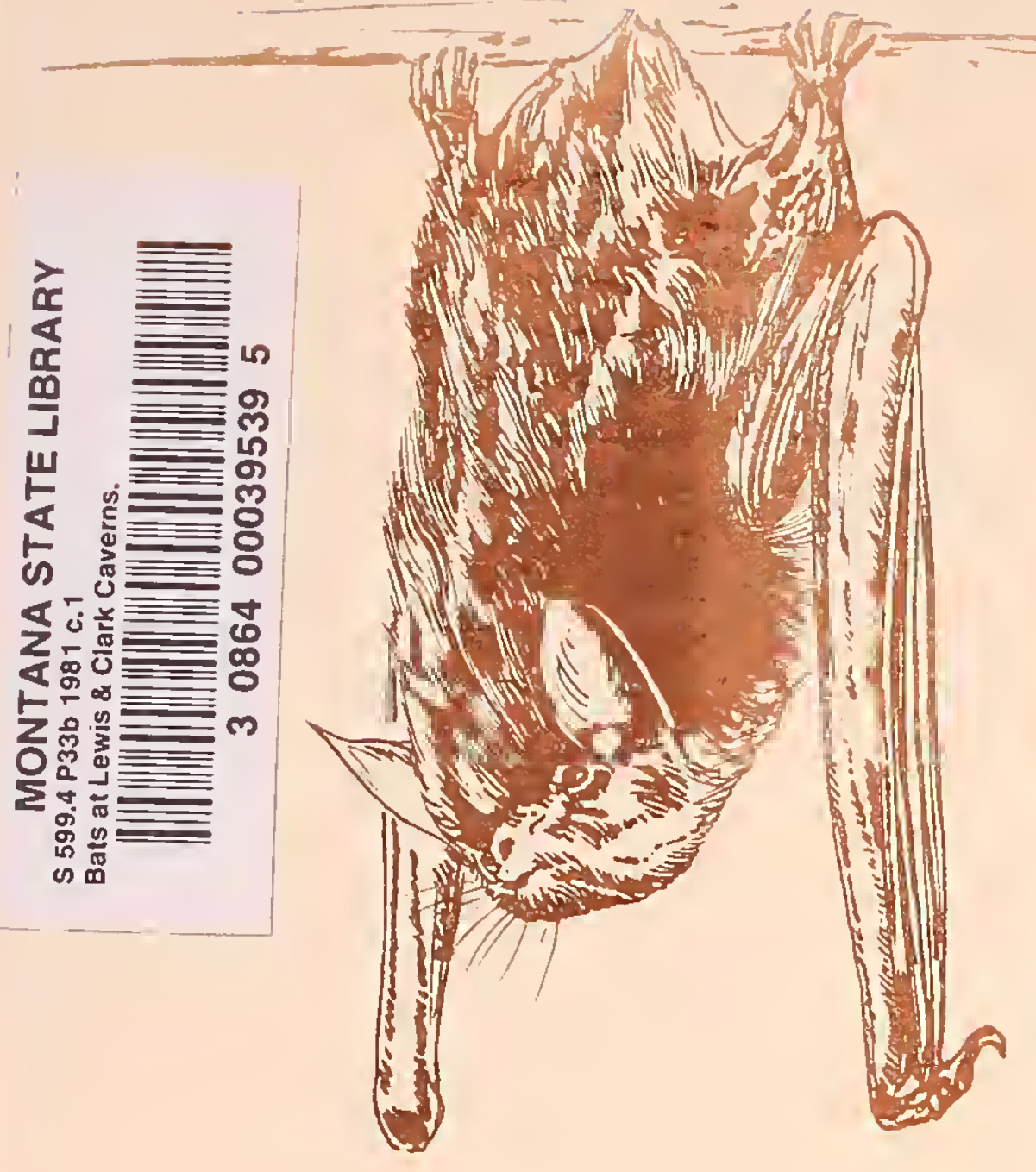

"Blind as a bat" is just one of many misnomers concerning bats. Bats see fairly well, but because they hunt at night and often live in caves, they have developed a superb sonar sys tem called "echolocation". This helps them to locate solid objects and aids in seizing insects as small as mosquitos as they fly. A bat will consume about 500 insects per hour when it is feeding.

Most people do not often encounter bats, and this has naturally led to many erroneous beliefs. For instance, bats do not tangle in people's hair. Neither do they kill their prey by sucking out their blood. The vampire bat (Desmodus rotundus) of South America makes a small thin cut with very sharp teeth and then laps up what little blood comes from the wound. The vampire bat is not found in the United States.

Rabies is carried by bats, but not to the extent generally believed. Most people who have been bitten by bats have disturbed or threatened them. As with any animal bite, bat bites should be promptly washed with soap and water and a physician should be called. It is important to remember that a bat flapping around on the ground or allowing people near it may not be a healthy bat and should be left alone.
The bat is the only mammal which has achieved true flight, and its body is specially developed so that this is possible. Bats have greatly elongated forelimbs and fingers which support the thin membranes that make up a bat's wings. Except for the short clawed thumbs which are free for grasping, the fingers are enclosed by these membranes. Extending back along the sides, the membranes are usually attached to the hind limbs at the ankles.

Another membrane stretches between the two hind limbs and, in most bats, this encloses most of the tail. This tail membrane also acts like a net to aid in catching insects while in flight. The hind toes are free and are used primarily for grasping when the bat rests in its typical head-down position.

Mating usually occurs in the late fall, but embryonic development does not begin until about February. The young are born alive in late spring with fully developed feet which allow them to cling to either their mother or their roost. The females nurse their young until they are about a month old. At this time, they are ready to take wing and become self-supporting. This rapid development is necessary because most bats are migratory and the young must be able to fly south to winter hibernation areas by autumn.

Females usually have just one baby each year, but some solitary species have from two to four. The higher reproduction rate is probably because of the much higher mortality rate suffered in their more exposed roosting places.

Solitary species such as the hoary bat and the silverhaired bat tend to live in trees. They roost hanging from branches or hide under loose bark.

Gregarious species tend to live in colonies and in the winter they hibernate in clusters. The reason for this is that as winter comes and temperatures drop, the bat's body temperature also decreases until it is just slightly warmer than the surrounding air. These tightly packed clusters of bats share their meager body heat and thus help to insure their survival through their hibernation.

Bats are able to store only a small amount of fat to sustain them through the winter. The little brown bat, for example, only stores about 5 grams of fat. For comparison, 5 grams of fat is smaller than a single cube of sugar. If disturbed

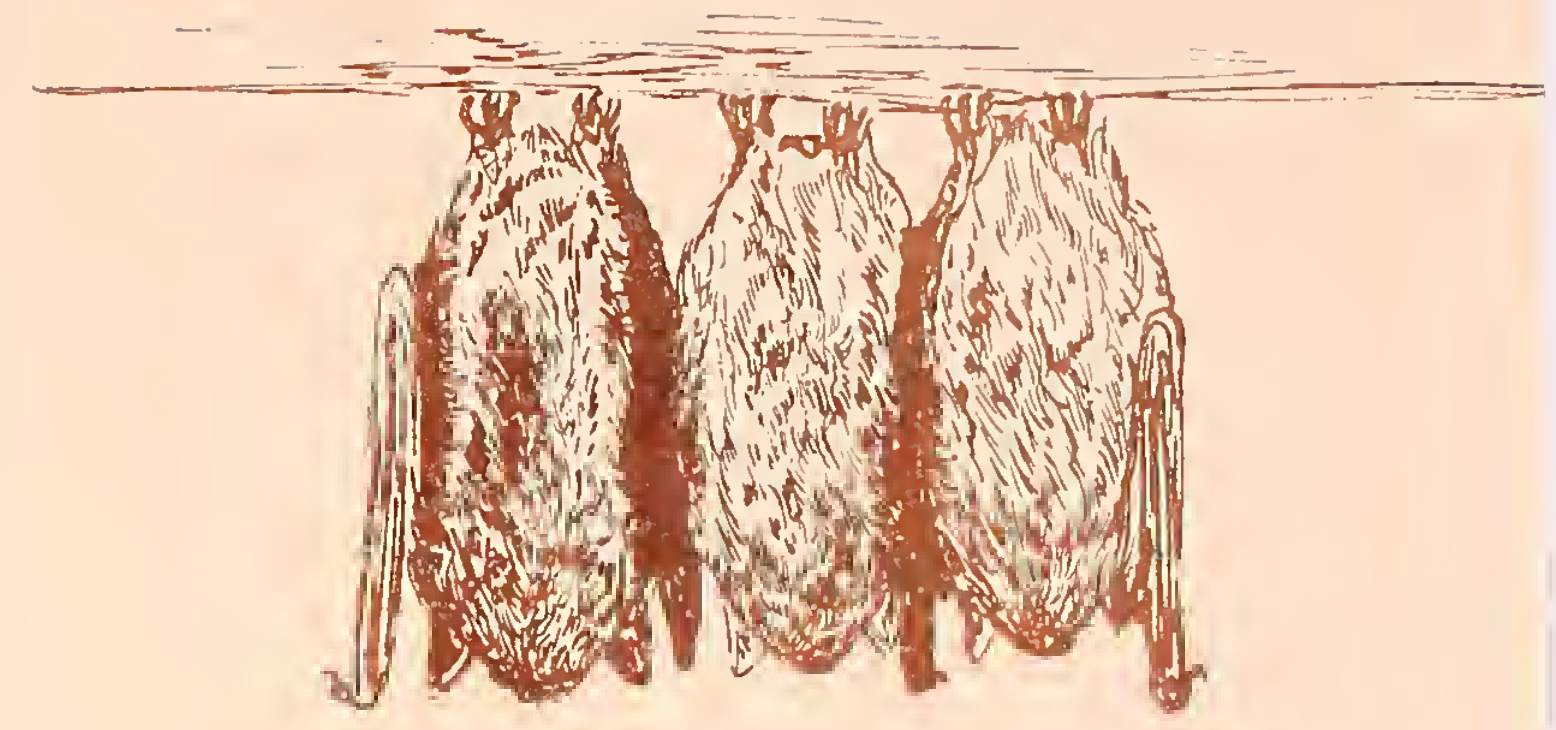

while hibernating, bats quickly rouse, using up stored nutrients. Since there are no insects to feed on in the winter to replenish their fat, they could die before spring.

In the last few years, scientists have noticed a serious decline in bat populations. Five species of bats are on the official U.S. Endangered Species List: the Indiana bat, the gray bat, the Ozark big-eared bat, the Virginia big-eared bat, and the Hawailan hoary bat. There are several major reasons for their decline.

One major reason, loss of habitat, can be the result of many things. Mining operations, urbanization, and construction of dams resulting in flooding of cave areas are major causes. Other reasons are disappearing mine shafts and hollow trees, as well as a decrease in roosting areas in modern buildings. These all contribute to a reduction of prime habitat areas.

Other major reasons for declining bat populations are vandalism and pesticides. Many people who don't understand that bats are important for controlling insect populations, kill them purposely. People exploring caves, mines, and old buildings may accidentally disturb them or kill them out of ignorance. Bats have also been severely affected by the widespread use of DDT and other insecticides which deplete and contaminate their food supply.

Bats are delicate and disturbing their roosts, particularly during hibernation, can kill them. Please help bats survive by NOT disturbing them. 


\section{Montana Species}

*Little Brown Bat

(Myotis lucifugus)

*Yuma Bat

(Myotis yumanensis)

Long-Eared Bat

(Myotis evotis)

California Brown Bat

(Myotis californicus)

Long-Legged Bat (Myotis volans)

Small Footed Bat (Least Bat) (Myotis leibii)

*Fringed Bat

(Myotis thysanodes)

Keen's Bat

(Myotis keenii)

Big Brown Bat

(Eptesicus fuscus)

Hoary Bat

(Lasiurus cinereus)

*Western Big-Eared Bat (Plecotus townsendii)

Pallid Bat (Antrozous pallidus)

Spotted Bat

(Euderma maculatum)

Silver-Haired Bat

(Lasionycteris noctivagans)

* These species are found in Lewis and Clark Caverns. The large clusters found in the Caverns in the summer are primarily western big-eared bats.

This brochure was prepared by the Parks Division of the Montana Department of Fish, Wildlife and Parks. We would like to express our thanks to the National Speleological Society for much of the information used. 


\section{Digitized by the Internet Archive in 2013}

http://archive.org/details/batsatlewisclark1981mont 


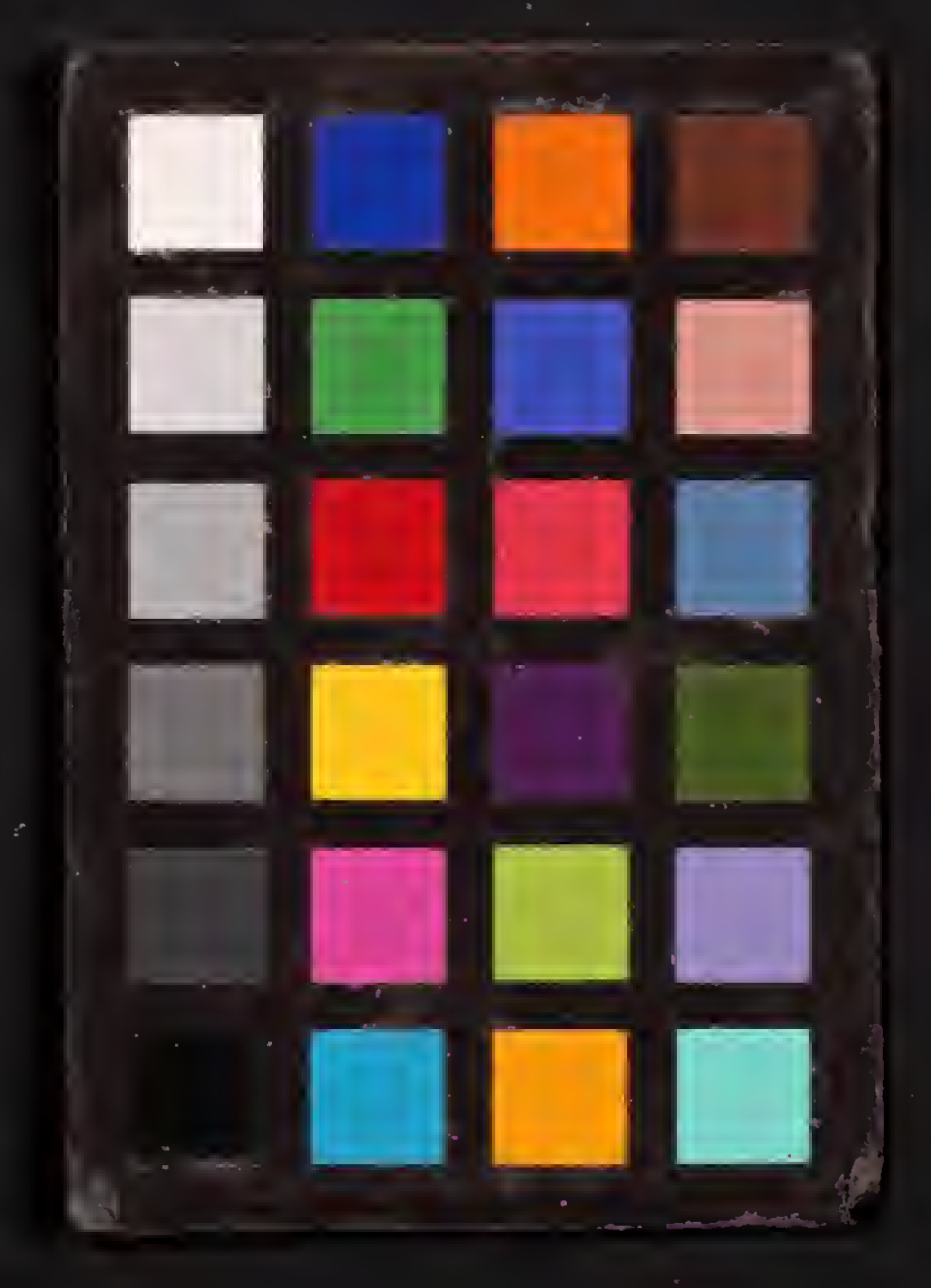

